\title{
Melanoma cell adhesion molecule stimulates yes-associated protein transcription by enhancing CREB activity via c-Jun/c-Fos in hepatocellular carcinoma cells
}

\author{
YONGXIA QIAO $^{1 *}$, YUNXIA QIAN $^{2 *}$, JIAYI WANG $^{3,4}$ and XUN TANG ${ }^{3}$ \\ ${ }^{1}$ School of Public Health, Shanghai Jiaotong University School of Medicine, Shanghai 200025; \\ ${ }^{2}$ Shanghai Yueke Bio-Technology Co., Ltd., Shanghai 201200; ${ }^{3}$ Department of Clinical Laboratory Medicine, \\ Shanghai Tenth People's Hospital of Tongji University, Shanghai 200072; \\ ${ }^{4}$ Advanced Institute of Translational Medicine, Tongji University, Shanghai 200092, P.R. China
}

Received March 10, 2015; Accepted March 22, 2016

DOI: $10.3892 / \mathrm{ol} .2016 .4442$

\begin{abstract}
Dysfunction of the yes-associated protein (YAP) signaling pathway has previously been associated with liver tumorigenesis. Recently, the membrane protein melanoma cell adhesion molecule (MCAM) was identified as a novel, hepatocellular carcinoma (HCC)-specific YAP target protein that promotes carcinogenesis in HCC. However, whether MCAM conversely regulates YAP remains unknown. The aim of the current study was to demonstrate whether and how MCAM regulates YAP in HCC cells. The present study demonstrated that MCAM has a positive effect on the regulation of YAP activity and expression. Mechanistically, MCAM stimulated YAP transcription through its downstream effector c-Jun/ c-Fos heterodimer. Gain and loss of function analysis by the present study indicated that c-Jun/c-Fos is capable of inducing cAMP response element-binding protein activation, which is a transcription factor that directly binds to the $Y A P$ promoter. Finally, it was identified that an impaired transformative phenotype in MCAM- or c-Jun/c-Fos-depleted HCC cells could be partially rescued by simultaneous overexpression of YAP, suggesting that YAP may function as a downstream effector of the MCAM-c-Jun/c-Fos signaling pathway. Collectively, a complete, positive, auto-regulatory loop was established by the present study, in which YAP is not only an upstream regulator, but also a downstream target of MCAM in HCC cells.
\end{abstract}

Correspondence to: Dr Jiayi Wang or Miss Xun Tang, Department of Clinical Laboratory Medicine, Shanghai Tenth People's Hospital of Tongji University, 301 Middle Yanchang Road, Shanghai 200072, P.R. China

E-mail: karajan2@163.com

E-mail: pangxun1990@qq.com

*Contributed equally

Key words: tumorigenesis, promoter, auto-regulatory loop, membrane protein, colony formation

\section{Introduction}

Hepatocellular carcinoma (HCC) is one of the most prevalent types of cancer worldwide (1) with an incidence rate of 10.1 cases per 100,000 individuals and a mortality rate of 9.5 cases per 100,000 individuals (2). Previously, dysfunction of the yes-associated protein (YAP) signaling pathway has been associated with liver tumorigenesis (3), and liver-specific YAP overexpression in transgenic mice resulted in tumor development (4). In addition, clinical studies have demonstrated that YAP is overexpressed and is a predictor of poor survival in patients with HCC (5). Due to the key role YAP is considered to play in liver tumorigenesis, it is extremely important to understand how this protein is regulated in HCC cells. A previous study established the role of cAMP response element-binding protein (CREB) in the upregulation of YAP in HCC cells, and identified a novel CREB binding site within the YAP promoter region (6). However, whether and how other proteins stimulate CREB-dependent transcription of YAP remains unclear.

Functioning as a cofactor, YAP increases the transcriptional activities of various transcription factors, including TEA domain (TEAD) family (7). Although several YAP target genes that operate in liver tumorigenesis have been gradually identified $(8,9)$, a large number remain unknown. Previously, the membrane protein, melanoma cell adhesion molecule (MCAM), was established as a novel HCC-specific YAP target (10). MCAM serum levels have been revealed to be specifically increased in HCC, indicating that MCAM serum levels may function as a specific tumor marker for HCC (10). In addition, it was demonstrated that MCAM is essential for $\mathrm{HCC}$ cell transformation and survival through the promotion of pro-tumorigenic c-Jun/c-Fos protein function (10). However, whether and how MCAM conversely regulates YAP function requires additional investigation.

The aim of the present study was to demonstrate whether MCAM regulates YAP in HCC cells and how this mechanism occurs. The present study demonstrated that MCAM is key in the stimulation of CREB-dependent transcription of YAP via the MCAM downstream effector c-Jun/c-Fos, and that YAP is a downstream effector of MCAM and c-Jun/c-Fos in the 
maintenance of the transformative phenotype of HCC cells. The present study established a complete, positive, auto-regulatory feedback loop, in which YAP is able to stimulate $M C A M$ gene transcription and MCAM conversely enhances $Y A P$ gene transcription.

\section{Materials and methods}

Cell culture and vectors. Human HCC Bel-7402, SMMC-7721 and Huh7 cells lines were purchased from Cell Bank of Type Culture Collection of Chinese Academy of Sciences (Shanghai, China) and cultured in Dulbecco's modified Eagle's medium (DMEM) supplemented with $10 \%$ fetal bovine serum (FBS) (Gibco ${ }^{\circledR}$; Thermo Fisher Scientific, Inc., Waltham, MA, USA) at $37^{\circ} \mathrm{C}$ in an incubator with $5 \% \mathrm{CO}_{2}$. Small hairpin RNAs (shRNAs) against MCAM (sh1 and sh2), c-Fos and c-Jun were purchased from GeneChem Co., Ltd. (Shanghai, China). The cDNA fragments encoding human MCAM were purchased from OriGene Technologies, Inc. (Beijing, China), and subcloned into a pcDNA3.1(+) vector (Invitrogen ${ }^{\mathrm{TM}}$; Thermo Fisher Scientific, Inc.) as previously described (10). The human c-Fos- and c-Jun-expressing vectors were purchased from OriGene Technologies, Inc.

Luciferase reporters containing either a CREB binding site (CREB-reporter) or a core $Y A P$ promoter region ( $Y A P$-promoter) were constructed as described previously $(6,11)$. The TEAD-Gal4/pUAS-Luc system was provided by Dr Junhao Mao (University of Massachusetts, Worcester, MA, USA) from the authors' previous study (12), and was used to indirectly test YAP activity, since YAP binds to the TEAD transcription factor. The YAP-expressing plasmids were constructed as described in a previous study (6). Briefly, the open reading frame of YAP was polymerase chain reaction (PCR)-amplified using the following primers: YAP, forward 5'-ATGCGCTAGCGATCCCGGGCA GCAGCCGCCGCCTC-3' and reverse 5'-ATGCGGCCGCCT ATAACCATGTAAGAAAGCTTTCTTTATC-3'. The cDNA of Bel-7402 cells was used as the template, and a pGIZ2a-lentiviral-based vector (provided by Dr Junhao Mao) was used as the backbone.

Immunofluorescence. Glass slides were seeded with Bel-7402 cells and were fixed by $4 \%$ paraformaldehyde (Scigen Ltd, Shanghai, China) for $15 \mathrm{~min}$, followed by washing with phosphate-buffered saline (PBS) for $5 \mathrm{~min}$ three times. Subsequently, the cells were incubated with blocking buffer [PBS solution containing 3\% FBS, 1\% goat serum (Jackson ImmunoResearch Laboratories, Inc., West Grove, PA, USA) and $0.1 \%$ Triton X-100 (Sangon Biotech Co., Ltd., Shanghai, China)] for $2 \mathrm{~h}$ at room temperature prior to incubation with primary antibodies overnight at $4^{\circ} \mathrm{C}$. The following primary antibodies were used at a dilution of 1:200, and were purchased from Cell Signaling Technology, Inc. (Danvers, MA, USA): Rabbit polyclonal anti-YAP (catalog no., 4912) and mouse monoclonal anti-FLAG (catalog no., 8146). The slides were then washed in PBS three times prior to incubation with Alexa Fluor ${ }^{\circledR}-488 / 555$ fluorescent conjugated secondary antibodies (mouse/rabbit monoclonal; catalog nos., 4408 and 4413, respectively; Cell Signaling Technology, Inc.) for $1 \mathrm{~h}$ in the dark. Subsequently, the slides were washed three times with PBS prior to being mounted with ProLong ${ }^{\circledR}$ Gold
Antifade Reagent with DAPI (Molecular Probes, Eugene, OR, USA). The sides were observed using a LSM 800 Confocal Microscope (Carl Zeiss AG, Oberkochen, Germany).

Western blot analysis. Cells were harvested and lysed using Western/IP buffer (Beyotime Institute of Biotechnology, Haimen, China) with protease inhibitor (Roche Diagnostics, Indianapolis, IN, USA). The supernatants of cell lysates were collected by centrifugation at $15,000 \mathrm{x} \mathrm{g}$ for $10 \mathrm{~min}$. The proteins was quantified by a BSA kit (Beyotime Institute of Biotechnology). Protein ( $30 \mu \mathrm{g}$ each sample) were resolved using $12 \%$ sodium dodecyl sulfate (SDS) polyacrylamide gel electrophoresis. The proteins were electrophoretically transferred onto Immobilon P Membrane (Bio-Rad Laboratories, Inc., Hercules, CA, USA). The blots were blocked with $5 \%$ non-fat milk in Tris-buffered saline (TBS; pH 7.4; Sangon Biotech Co., Ltd.) for $1 \mathrm{~h}$ at room temperature and subsequently incubated with primary antibodies at $4^{\circ} \mathrm{C}$ overnight. The primary antibodies were used at a dilution of 1:1,000 and were as follows: Rabbit monoclonal anti-MCAM (catalog no., 2505-1) rabbit monoclonal anti-YAP (catalog no., 2060-1), and rabbit monoclonal anti-c-Jun (catalog no., 1254-1) (Epitomics, Burlingame, CA, USA); mouse monoclonal anti-FLAG (catalog no., F3165; Sigma-Aldrich, St. Louis, MO, USA); rabbit polyclonal anti-FLAG (catalog no., 2368), rabbit monoclonal anti-CREB (catalog no., 9197), rabbit monoclonal anti-phospho-CREB (catalog no., 9198), rabbit monoclonal anti-glyceraldehyde 3-phosphate dehydrogenase (GAPDH; catalog no., 5174) and rabbit monoclonal anti-c-Fos (catalog no., 2250) (Cell Signaling Technology, Inc.). The membranes were washed with TBS and incubated with secondary antibodies conjugated with horseradish peroxidase (catalog no., 7074 and 7076; Cell Signalling Technology, Inc.) for $1 \mathrm{~h}$ at room temperature. After washing with TBS, the membranes were visualized using Pierce ECL Western Blotting Substrate (Pierce ${ }^{\mathrm{TM}}$; Thermo Fisher Scientific, Inc.) and recorded on X-ray films. YAP protein levels were normalized to those of GAPDH and p-CREB levels were normalized to those of total CREB. Densitometry of the blots was performed using ImageJ version 1.47 software (National Institutes of Health, Bethesda, MD, USA).

Luciferase reporter analysis. Luciferase reporter plasmids were transiently transfected into Bel-7402, SMMC-7721 or Huh7 cells using Lipofectamine ${ }^{\circledR} 2000$ (Invitrogen $^{\mathrm{TM}}$; Thermo Fisher Scientific, Inc.). pRL-TK (Promega Corporation, Madison, WI, USA) was co-transfected as an internal control to calibrate transfection efficiency. Following additional cultivation for $24 \mathrm{~h}$, the transfected cells were harvested and lysed used $1 \mathrm{X}$ passive lysis buffer, centrifuged at 15,000 x g for $10 \mathrm{~min}$ and the pellet subjected to luciferase assay. Luciferase activity was measured as chemiluminescence using a plate reader $(\Sigma 960$; Metertech, Inc., Taipei, Taiwan) and the Dual-Luciferase ${ }^{\circledR}$ Reporter Assay System (Promega Corporation), according to the manufacturer's protocol. Firefly luciferase activities were normalized to those from Renilla.

Reverse transcription-quantitative PCR ( $q P C R)$. Total RNA from Bel-7402, SMMC-7721 and Huh7 cells was extracted using TRIzol Reagent (Invitrogen ${ }^{\mathrm{TM}}$; Thermo Fisher Scientific, Inc.). In total, $20 \mathrm{ml}$ reaction mixture was used to reverse 

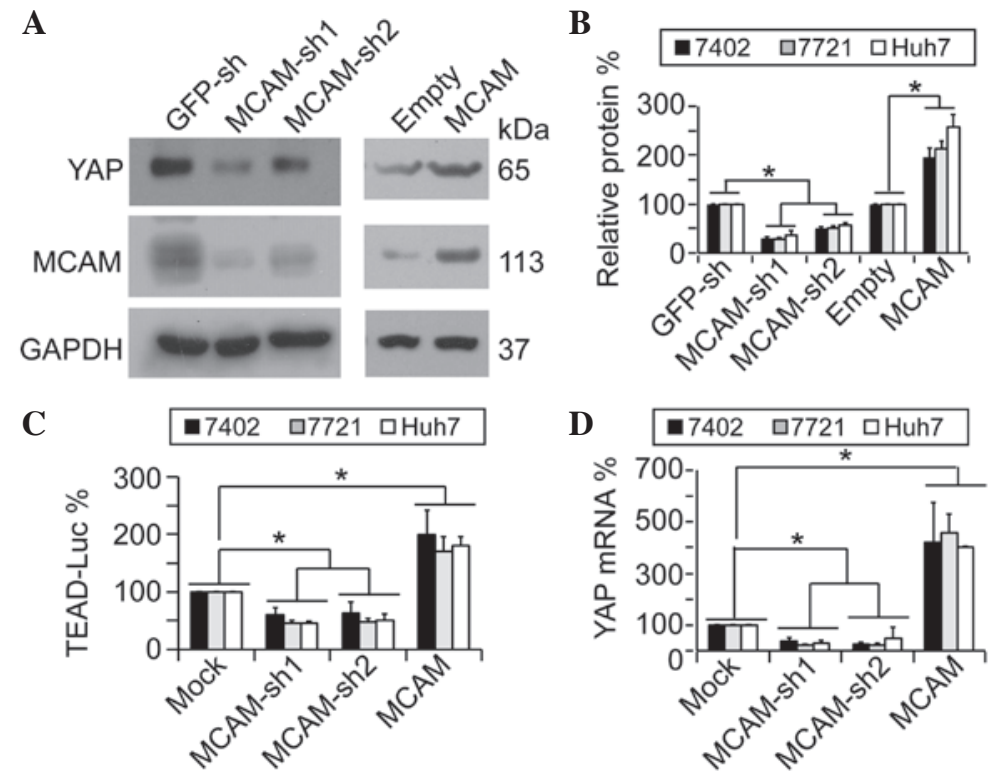

Figure 1. YAP is positively regulated by MCAM. (A and B) Representative western blotting images and quantification of western blots of YAP and MCAM in human HCC Bel-7402 cells under various treatments. The results demonstrated that MCAM regulated YAP protein expression. (C) MCAM affected YAP activity. The pUAS-Luc-TEAD-Gal4 luciferase reporter systems were transfected into human HCC Bel-7402, SMMC-7721 or Huh7 cells with or without MCAM-knockdown or overexpression. (D) MCAM regulated YAP mRNA levels. mRNA levels of YAP were measured by quantitative polymerase chain reaction in Bel-7402, SMMC-7721 or Huh7 cells with or without MCAM-knockdown or overexpression. Data are presented as the mean \pm standard deviation of three independent experiments. Mock and GFP-sh groups were arbitrarily set to $100 \%$. ${ }^{*} \mathrm{P}<0.05$. YAP, yes-associated protein; MCAM, melanoma cell adhesion molecule; GAPDH, glyceraldehyde 3-phosphate dehydrogenase; sh, small hairpin; GFP, green fluorescent protein; TEAD, TEA domain; Luc, luciferase; HCC, hepatocellular carcinoma; MOCK, control cells without treatment.

transcribe $1 \mathrm{mg}$ total RNA to cDNA using PrimeScript RT Reagent kit (Takara, Bio, Inc., Otsu, Japan). qPCR was performed on the cDNA using primers specific for YAP. The primers were purchased from BGI Shenzhen (Shenzhen, China) and were as follows: YAP, forward 5'-CCTCGTTTT GCCATGAACCAG-3' and reverse 5'-GTTCTTGCTGTT TCAGCCGCAG-3'; GAPDH, forward 5'-ATCATCCCTGCC TCTACTGG-3' and reverse 5'-GTCAGGTCCACCACTGAC AC-3'. SYBR Green Mix kit (Takara Bio, Inc.) was used for the qPCR. The conditions for qPCR were as follows: Activation at $94^{\circ} \mathrm{C}$ for $5 \mathrm{~min}$, and then 40 cycles of denaturation at $94^{\circ} \mathrm{C}$ for $30 \mathrm{sec}$ and combined annealing and extension at $60^{\circ} \mathrm{C}$ for 30 sec. The RT-qPCR was performed on an ABI 7500 Real-time PCR System (Applied Biosystems ${ }^{\circledR}$; Thermo Fisher Scientific, Inc.). The fluorescence of each sample was determined following every cycle. Relative expression levels were calculated as the ratios normalized against those of GAPDH, using the $\Delta \mathrm{Cq}$ method. The $\Delta \mathrm{Cq}$ value was determined by subtracting the GAPDH $\Delta \mathrm{Cq}$ value from the YAP gene $\Delta \mathrm{Cq}$ value. The $\Delta \mathrm{Cq}$ of the stimulated cells ( $\Delta \mathrm{Cqt})$ was subtracted from the $\Delta \mathrm{Cq}$ of the untreated cells ( $\Delta \mathrm{Cqu}$ ) as follows: $\Delta \Delta \mathrm{Cq}=\Delta \mathrm{Cqt}-\Delta \mathrm{Cqu}$. The expression level for the YAP gene in the stimulated cells compared with the level in the untreated cells was calculated as follows: $x$-fold of unstimulated control $=2^{-\Delta \Delta C q}(13)$.

Chromatin immunoprecipitation (ChIP). ChIP assays were performed with the ChIP-IT ${ }^{\circledR}$ Express kit (catalog no., 53008; Active Motif, Carlsbad, CA, USA), according to the manufacturer's protocol. Briefly, $1 \times 10^{7}$ cells were fixed with $1 \%$ formaldehyde, washed with cold PBS and lysed in 1X lysis buffer. Nuclei were sonicated (JY92-IIN; Scientz Biotech Co.,
Ltd., Ningbo, China) to shear DNA, and the lysates were pelleted and precleared using $\mathrm{A} / \mathrm{G}$ beads (Novex ${ }^{\circledR}$; Thermo Fisher Scientific, Inc.). The beads were separated by centrifugation at $15,000 \mathrm{x} \mathrm{g}$ for $20 \mathrm{~min}$ and the protein-DNA complexes were incubated with $3 \mu \mathrm{g}$ rabbit monoclonal anti-CREB antibody and $10 \mu \mathrm{l}$ protein $\mathrm{A} / \mathrm{G}$ beads in a total reaction mixture of $200 \mu \mathrm{l}$ overnight at $4^{\circ} \mathrm{C}$. The protein-DNA complexes were eluted in $1 \% \mathrm{SDS} / 0.1 \mathrm{M} \mathrm{NaHCO}_{3}$ and cross-links were reversed at $65^{\circ} \mathrm{C}$. Finally, DNA was subjected to qPCR analysis following recovery using a SYBR Green Mix kit (Takara, Bio, Inc.) and an ABI 7500 Real-time PCR System (Applied Biosystems $^{\circledR}$; Thermo Fisher Scientific, Inc.) with the following cycling conditions: $94^{\circ} \mathrm{C}$ for $5 \mathrm{~min}$, then 40 cycles of $94^{\circ} \mathrm{C}$ for $30 \mathrm{sec}$, and $60^{\circ} \mathrm{C}$ for $30 \mathrm{sec}$. The primers were purchased from BGI Shenzhen and were used for detection of CREB binding on the YAP promoter (R1 and R2), as follows: R1, forward 5'-CCAACAACTATGAGGTAGGTG-3' and reverse 5'-GAG TTTTGAGTTCAGGTCCCAGTTC-3'; R2, forward 5'-GCA GACTAACAGATAAGTGAAAC-3' and reverse 5'-TCCGCT CCGCTCGGCCCCTTTTC-3'. The fluorescence of each sample was determined following every cycle. Relative enrichments of CREB binding within the YAP promoter were calculated as the ratios normalized against those of the input sample, using the $\Delta \mathrm{Cq}$ method. The $\Delta \mathrm{Cq}$ value was determined by subtracting the input sample $\Delta \mathrm{Cq}$ value from the CREB ChIP sample $\Delta \mathrm{Cq}$ value. The percentage of input (\%) was calculated as follows: $2^{-\Delta \Delta \mathrm{Cq}}$ x $100 \%$ (11).

Soft-agar assays. Anchorage-independent soft-agar growth assays were performed as previously described (6). Briefly, control (Bel-7402, SMMC-7721 or Huh7 without treatment), Bel-7402, SMMC-7721 or Huh7 cells with MCAM, c-Jun 
A

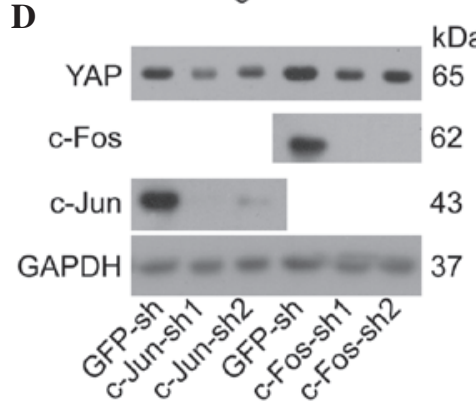

B

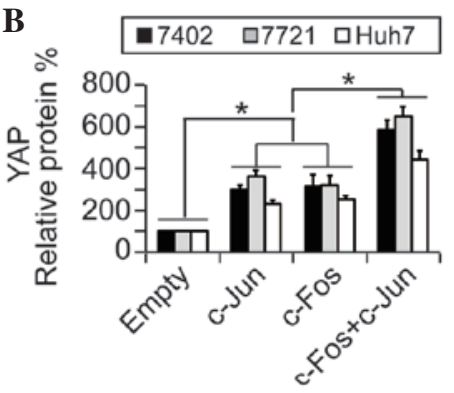

E
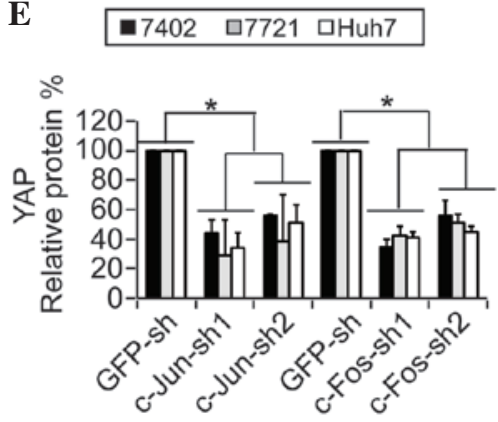

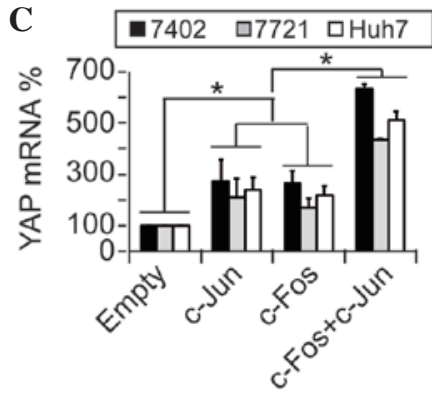

F
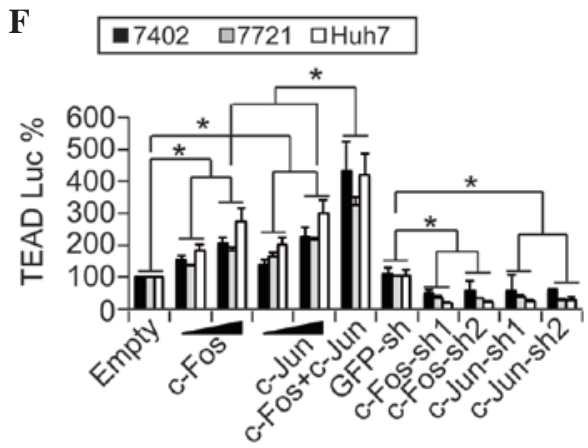

G

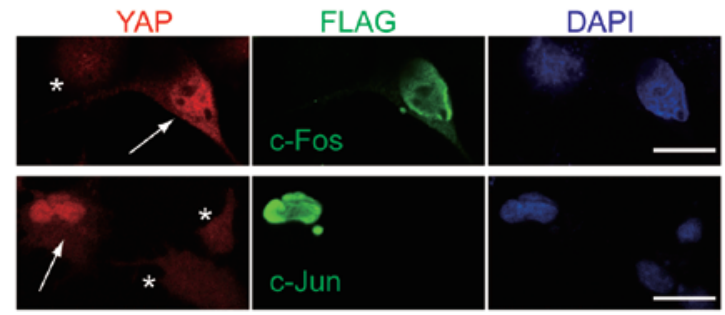

Figure 2. Activity and expression of YAP is regulated by c-Jun/c-Fos. (A and B) Overexpression of c-Jun/c-Fos upregulated YAP protein expression as shown by (A) representative western blotting images and (B) quantification of YAP, c-Jun and c-Fos in human HCC Bel-7402 cells with or without c-Jun/c-Fos overexpression. (C) c-Jun/c-Fos upregulated YAP mRNA levels. (D and E) Knockdown of c-Jun/c-Fos reduced YAP protein expression as shown by (D) representative western blotting images and (E) quantification of YAP, c-Fos and c-Jun in Bel-7402 cells with or without c-Jun/c-Fos-knockdown. (F) YAP activity was regulated by c-Jun/c-Fos. The pUAS-Luc-TEAD-Gal4 luciferase reporter systems were transfected into human HCC Bel-7402, SMMC-7721 or Huh7 cells with or without c-Jun/c-Fos-knockdown or overexpression (transfected with 0.5-1.5 $\mu \mathrm{g}$ plasmids). (G) Endogenous cellular YAP expression was increased by overexpression of c-Jun/c-Fos, as measured by immunofluorescence in Bel-7402 cells transfected with exogenous c-Fos-FLAG or c-Jun-FLAG-expressing plasmids. Arrows indicate cells with c-Jun/c-Fos transfection, while asterisks indicate cells without successful transfection. Scale bar, $20 \mu \mathrm{m}$. Data are presented as the mean \pm standard deviation of three independent experiments. Empty and GFP-sh groups were arbitrarily set to $100 \%$. ${ }^{*}<0.05$. YAP, yes-associated protein; GAPDH, glyceraldehyde 3-phosphate dehydrogenase; sh, small hairpin; GFP, green fluorescent protein; TEAD, TEA domain; Luc, luciferase; DAPI, 4',6-diamidino-2-phenylindole; HCC, hepatocellular carcinoma.

or c-Fos knocked-down, using shRNAs, in the presence or absence of overexpression of YAP were seeded onto 6-well agar plates at a density of $5 \times 10^{3}$ cells/well and maintained in DMEM supplemented with $1 \mu \mathrm{g} / \mathrm{ml}$ puromycin (Mediatech, Inc., Manassas, VA, USA) for 4 weeks until foci were evident. Colonies were subsequently counted.

Statistical analysis. Student's t-test was used to examine the differences between groups using STATA version 11.0 software (StataCorp LP, College Station, TX, USA). P<0.05 was considered to indicate a statistically significant difference. Results are expressed as the mean \pm standard deviation of three independent experiments.

\section{Results}

MCAM conversely regulates YAP. To investigate whether MCAM conversely regulates YAP, YAP protein expression was analyzed prior to and following knockdown or overexpression of MCAM. The results demonstrated that YAP protein levels were downregulated by MCAM-knockdown through two independent MCAM shRNAs (sh1 and sh2, respectively), whilst overexpression of MCAM resulted in upregulated YAP protein levels (Fig. 1A and B). Since TEAD transcription factors mediate YAP-dependent transcriptional activity (7), the present study utilized a previously reported pUAS-Luc-TEAD-Gal4 system (12) to indirectly test YAP activity prior to and following knockdown or overexpression of MCAM. It was demonstrated that TEAD-controlled downstream luciferase activities could be reduced significantly by MCAM-sh1 and -sh2, and could be induced by ectopically-expressed MCAM (Fig. 1C), thus suggesting that MCAM also induces YAP activity. In addition, it was observed that MCAM had a similar effect on YAP mRNA as that on YAP protein (Fig. 1D), indicating that MCAM regulates YAP expression primarily through its effect on YAP transcription.

YAP is regulated by $c$-Jun/c-Fos. It was previously reported that c-Jun/c-Fos proteins function as key downstream effectors of MCAM in the maintenance of a transformative phenotype 
A

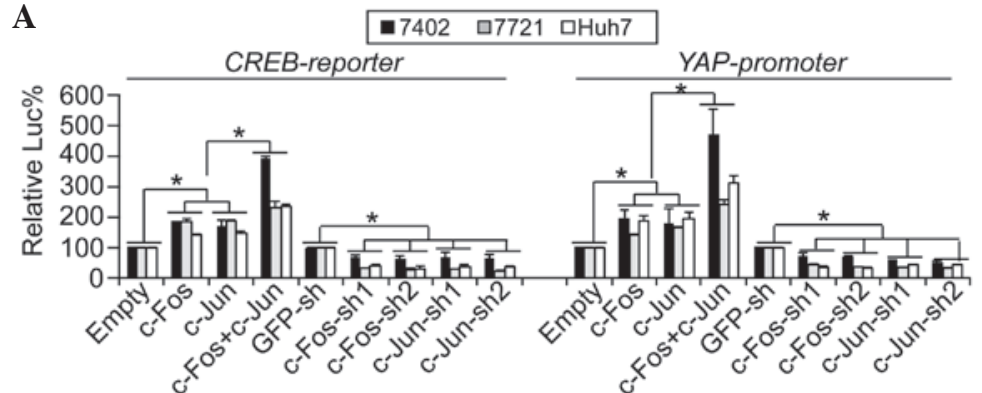

B



C

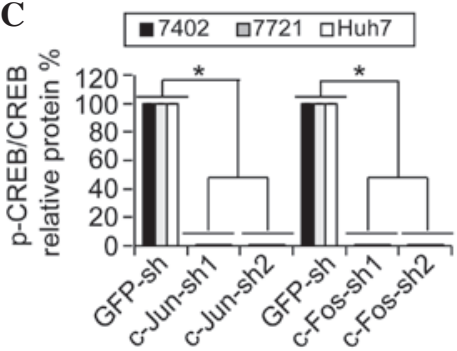

D

C-Fos-FLAG : + C-Jun-FLAG - - + $\mathrm{kDa}$ $\mathrm{p}-\mathrm{CREB} \equiv=43$

CREB --43

FLAG $\quad 62$

GAPDH $=37$
E

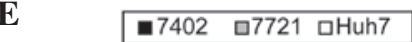

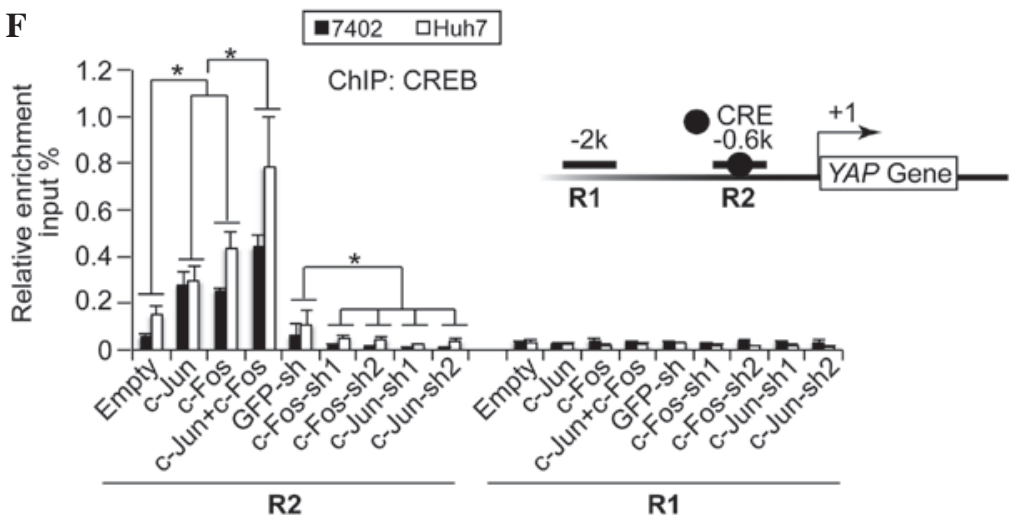

Figure 3. YAP transcription is regulated by c-Jun/c-Fos via CREB. (A) c-Jun/c-Fos upregulated CREB-dependent transcription and YAP promoter activities. Luciferase reporter plasmids, containing a CREB binding site (CREB-reporter) or YAP promoter regions (YAP-promoter), were co-transfected with pRL-TK-Renilla reporter plasmids into human hepatocellular carcinoma Bel-7402, SMMC-7721 or Huh7 cells with various treatments. (B and C) Knockdown of c-Jun/c-Fos reduced CREB phosphorylation as shown by (B) representative western blotting images and (C) quantification of p-CREB and CREB in Bel-7402 cells with or without c-Jun/c-Fos-knockdown. Even with a long exposure, p-CREB was hardly detected. (D and E) Overexpression of c-Jun/c-Fos facilitated phosphorylation of CREB as shown by (D) representative western blotting images and (E) quantification of p-CREB and CREB in Bel-7402 cells with or without c-Jun/c-Fos overexpression. (F) c-Jun/c-Fos stimulated CREB binding to the YAP gene promoter region. ChIP was performed using anti-CREB antibodies in Bel-7402 and Huh7 cells under various treatments, using primer sets encompassing R2 and R1 regions within the YAP promoter. Insert shows YAP promoter region, with the black circle indicating a CRE site. The relative enrichments of CREB were normalized to the percentage of the input chromosomal DNA. Data are presented as the mean \pm standard deviation of three independent experiments. Empty and GFP-sh groups were arbitrarily set to $100 \%$. "P<0.05. CREB, cAMP response element-binding protein; p-CREB, phospho-CREB; YAP, yes-associated protein; Luc, luciferase; GAPDH, glyceraldehyde 3-phosphate dehydrogenase; sh, small hairpin; GFP, green fluorescent protein; ChIP, chromatin immunoprecipitation; CRE, cAMP response element.

in HCC cells (10). Therefore, the present study investigated whether MCAM regulates YAP through c-Jun/c-Fos. The results demonstrated that the overexpression of c-Jun and c-Fos resulted in a significant upregulation of YAP protein expression, and these effects were synergized by a co-overexpression of c-Jun and c-Fos (Fig. $2 \mathrm{~A}$ and $\mathrm{B} ; \mathrm{P}<0.05$ ). Subsequently, whether c-Jun/c-Fos regulates YAP mRNA levels was analyzes, and it was observed that YAP mRNA levels were also upregulated by c-Jun/c-Fos (Fig. 2C). By contrast, knocking down c-Jun and c-Fos led to a significant downregulation of YAP protein (Fig. 2D and E; P<0.05). Data from the pUAS-Luc-TEAD-Gal4 system indicated that TEAD-controlled downstream luciferase activities may be upregulated in a dose-dependent manner by transfection of increasing concentrations of c-Jun/c-Fos, in addition to demonstrating that the simultaneous effects of c-Jun and
c-Fos were synergic (Fig. 2F). Conversely, TEAD-controlled downstream luciferase activities could be downregulated by c-Jun/c-Fos-knockdown (Fig. 2F). Furthermore, immunofluorescence assays demonstrated that YAP expression levels were increased in Bel-7402 cells with a successful transfection of c-Jun/c-Fos-expressing plasmids compared with untransfected control cells (Fig. 2G). These results suggest that the MCAM downstream effectors, c-Jun/c-Fos, may be critical in the MCAM-induced upregulation of YAP.

YAP transcription is upregulated by c-Jun/c-Fos via activation of CREB. The present study demonstrated that c-Jun/c-Fos upregulated YAP by enhancing its transcription, and it was also reported previously that CREB is critical in the transcription of YAP (6). Therefore, the present study hypothesized that c-Jun/c-Fos may upregulate the transcription of YAP via 
A

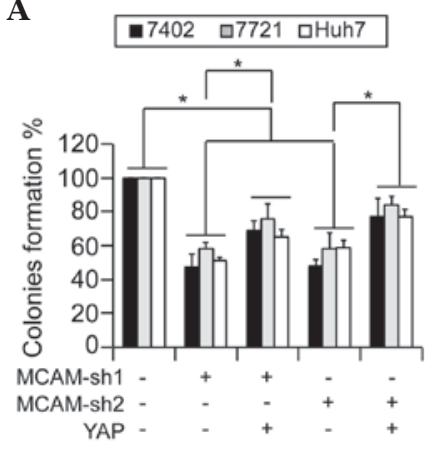

B

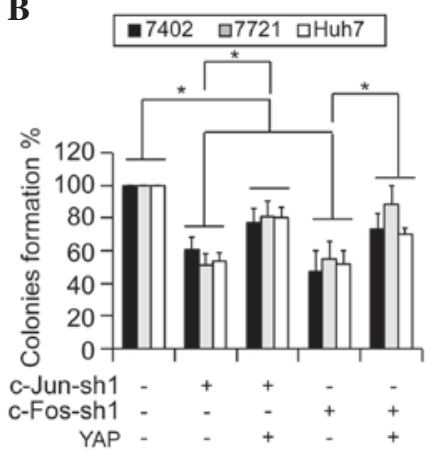

C

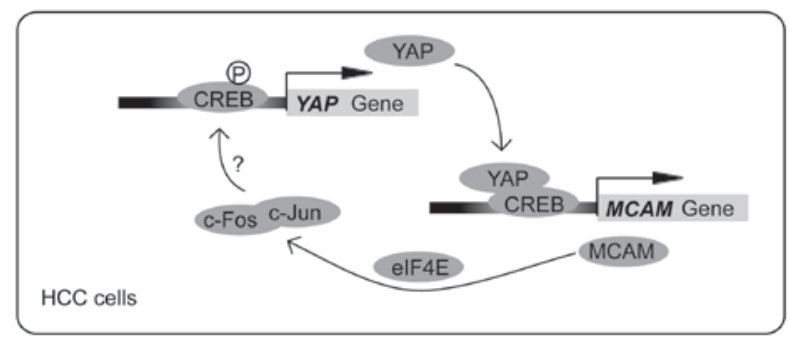

Figure 4. YAP functions as a downstream effector of MCAM and c-Jun/c-Fos during tumorigenesis. Relative anchorage-independent soft-agar colony formation assays in human HCC Bel-7402, SMMC-7721 and Huh7 cells expressing shRNA constructs against either (A) MCAM, (B) c-Jun or c-Fos with or without ectopic expression of YAP. Mock group was arbitrarily set to $100 \%$. " $\mathrm{P}<0.05$. Data are presented as the mean \pm standard deviation of three independent experiments. (C) Representation of the possible positive, auto-regulatory feedback loop between MCAM and YAP in HCC cells. MCAM, melanoma cell adhesion molecule; YAP, yes-associated protein; sh, small hairpin; CREB, cAMP response element-binding protein; HCC, human hepatocellular carcinoma.

CREB. To investigate this, CREB-dependent transcriptional activities were analyzed using a luciferase reporter containing a CREB binding site (CREB-reporter). It was demonstrated that overexpression of either c-Jun or c-Fos resulted in upregulation of CREB-dependent luciferase activities, and such effects could be synergized by simultaneous overexpression of c-Jun and c-Fos (Fig. 3A). By contrast, c-Jun/c-Fos-knockdown resulted in significantly reduced CREB-dependent luciferase activities (Fig. 3A; $\mathrm{P}<0.05$ ). In a similar manner to the CREB-reporter, c-Jun/c-Fos upregulated luciferase activities from the reporter containing the core $Y A P$ promoter regions (YAP-promoter) (Fig. 3A).

As the phosphorylation of CREB at Ser-133 (p-CREB) is a characteristic of CREB activation (14), the present study investigated whether c-Jun/c-Fos affects the phosphorylation of CREB. The results demonstrated that c-Jun/c-Fos-knockdown significantly downregulated p-CREB (Fig. $3 \mathrm{~B}$ and $\mathrm{C}$; $\mathrm{P}<0.05$ ), whilst overexpression of c-Jun/c-Fos resulted in significantly upregulated levels of p-CREB (Fig. 3D and E; P<0.05). These results suggest that c-Jun/c-Fos stimulates activation of CREB.

It was subsequently investigated whether c-Jun/c-Fos could directly stimulate recruitment of CREB onto the YAP promoter. It was observed that the co-overexpression of c-Jun and c-Fos induced a significant enrichment of $\mathrm{CREB}$ onto the $\mathrm{R} 2$ region, which contains a known CREB binding site, of the YAP promoter compared with single overexpression of c-Jun and c-Fos (Fig. 3F). However, no CREB enrichment was detected at the $\mathrm{R} 1$ region (Fig. 3F), which is a CREB-unrelated region. By contrast, c-Jun- and c-Fos-knockdown reduced recruitment of CREB onto the YAP promoter at the $\mathrm{R} 2$ region, whereas this had no effect on the $\mathrm{R} 1$ region (Fig. 3F). These results indicate that c-Jun/c-Fos stimulates YAP transcription through the induction of CREB recruitment onto the YAP promoter.
Associations between MCAM, c-Jun/c-Fos and YAP are critical in liver tumorigenesis. The present study analyzed whether the associations between MCAM, c-Jun/c-Fos and YAP are important for the maintenance of a transformative phenotype in HCC cells. It was observed that knockdown of MCAM significantly impaired the ability of the Bel-7402, SMMC-7721 and Huh7 cells to form colonies in soft-agar, which could be partially reversed by simultaneous overexpression of YAP (Fig. 4A). Furthermore, c-Jun/c-Fos-knockdown induced downregulation of colony formation, which could also be partially rescued by overexpression of YAP (Fig. 4B). These results indicate that YAP is a potential downstream target of the MCAM-c-Jun/c-Fos signaling cascade, and the associations between MCAM, c-Jun/c-Fos and YAP may be critical during liver tumorigenesis.

\section{Discussion}

Recently, MCAM was identified as a direct target of YAP in HCC cells (10). Additionally, in the present study, it was demonstrated that YAP expression could be conversely regulated by MCAM through its downstream effector, c-Jun/c-Fos. c-Jun and c-Fos proteins are able to form heterodimers, which are capable of promoting proliferation and neoplastic transformation (15). Notably, c-Jun/c-Fos dimers bind with high affinity to cAMP response elements (CREs), which are CREB binding sites (15). Furthermore, CREB is able to efficiently form a heterodimeric complex with c-Jun, and the interaction between the two proteins increases the repertoire of possible regulatory complexes that are important in the regulation of transcription (16). Although no previous study has yet established a direct interaction between CREB and c-Fos, the present study observed that the synergic effects 
of c-Fos and c-Jun resulted in the recruitment of CREB onto the $Y A P$ promoter and the upregulation of YAP expression. Therefore, the present study hypothesizes that c-Jun, c-Fos and CREB may scaffold into a complex, and the close interactions between these three proteins may be fundamental for the transcriptional regulation of certain genes, including YAP, in the promotion of tumorigenesis.

The present study hypothesizes that the critical step during the regulation of YAP by MCAM is the phosphorylation of CREB at Ser-133, which is facilitated by c-Jun/c-Fos. Ser-133 may be phosphorylated by various kinases, including protein kinase B, ribosomal protein S6 kinase, mitogen- and stress-activated protein kinase and protein kinase C $(17,18)$, with its phosphorylation promoting the activation of CREB $(19,20)$. Despite no direct evidence to suggest that c-Jun/c-Fos proteins possess a kinase function, c-Jun/c-Fos are able to be phosphorylated by other kinases. It has been demonstrated that constitutive activation of the extracellular-signal-regulated kinase (ERK) signaling pathway activates c-Jun by phosphorylation, and thus contributes to tumorigenesis by increasing cell proliferation (21). Furthermore, ERK signaling catalyzes the phosphorylation of c-Fos at Ser-374 $(22,23)$. Notably, it was reported that ERK phosphorylation is associated with increased phosphorylation of CREB (24). As ERK functions as a signal transmitter between the cytoplasm and nucleus, the overexpression of membrane-bound MCAM in HCC may attract ERK to translocate from the cytoplasm to CRE sites in the nucleus. With the aid of c-Jun/c-Fos, ERK may add a phosphate group onto CREB and consequently stimulate CREB-dependent YAP transcription. However, further investigation is required to fully elucidate the associations between MCAM, c-Jun/c-Fos and CREB.

In conclusion, the current study established a complete, positive, auto-regulatory loop between MCAM and YAP. Inhibition of this loop may serve as a possible target for the treatment of HCC.

\section{Acknowledgements}

The present study was supported by the National Natural Science Foundation of China (Beijing, China; grant no. 81301689), Yangfan Project of the Shanghai Committee of Science and Technology (Shanghai, China; grant no. 14YF1412300), Young College Teachers' Training Scheme of Shanghai (Shanghai, China; grant no. ZZjdyx13007), Outstanding Youth Training Program of Tongji University (Shanghai, China; grant no. 1501219080) and Shanghai Tenth People's Hospital Climbing Training Program (Shanghai, China; grant no. 04.01.13024).

\section{References}

1. Farazi PA and DePinho RA: Hepatocellular carcinoma pathogenesis: From genes to environment. Nat Rev Cancer 6: 674-687, 2006.

2. Ferlay J, Soerjomataram I, Dikshit R, Eser S, Mathers C, Rebelo M, Parkin DM, Forman D and Bray F: Cancer incidence and mortality worldwide: Sources, methods and major patterns in GLOBOCAN 2012. Int J Cancer 36: E359-E386, 2015.
3. Zeng Q and Hong W: The emerging role of the hippo pathway in cell contact inhibition, organ size control and cancer development in mammals. Cancer Cell 13: 188-192, 2008.

4. Dong J, Feldmann G, Huang J, Wu S, Zhang N, Comerford SA, Gayyed MF, Anders RA, Maitra A and Pan D: Elucidation of a universal size-control mechanism in Drosophila and mammals. Cell 130: 1120-1133, 2007.

5. Xu MZ, Yao TJ, Lee NP, Ng IO, Chan YT, Zender L, Lowe SW, Poon RT and Luk JM: Yes-associated protein is an independent prognostic marker in hepatocellular carcinoma. Cancer 115: 4576-4585, 2009.

6. Wang J, Ma L, Weng W, Qiao Y, Zhang Y, He J, Wang H, Xiao W, Li L, Chu Q, et al: Mutual interaction between YAP and CREB promotes tumorigenesis in liver cancer. Hepatology 58: 1011-1020, 2013.

7. Zhao B, Ye X, Yu J, Li L, Li W, Li S, Yu J, Lin JD, Wang CY, Chinnaiyan AM, et al: TEAD mediates YAP-dependent gene induction and growth control. Genes Dev 22: 1962-1971, 2008.

8. Urtasun R, Latasa MU, Demartis MI, Balzani S, Goñi S, Garcia-Irigoyen O, Elizalde M, Azcona M, Pascale RM, Feo $\mathrm{F}$, et al: Connective tissue growth factor autocriny in human hepatocellular carcinoma: Oncogenic role and regulation by epidermal growth factor receptor/yes-associated protein-mediated activation. Hepatology 54: 2149-2158, 2011.

9. Li H, Wolfe A, Septer S, Edwards G, Zhong X, Abdulkarim AB, Ranganathan S and Apte U: Deregulation of Hippo kinase signalling in human hepatic malignancies. Liver Int 32: 38-47, 2012.

10. Wang J, Tang X, Weng W, Qiao Y, Lin J, Liu W, Liu R, Ma L, $\mathrm{Yu}$ W, Yu Y, et al: The membrane protein melanoma cell adhesion molecule (MCAM) is a novel tumor marker that stimulates tumorigenesis in hepatocellular carcinoma. Oncogene 34: 5781-5795, 2015

11. Wang J, Liu X, Wu H, Ni P, Gu Z, Qiao Y, Chen N, Sun F and Fan Q: CREB up-regulates long non-coding RNA, HULC expression through interaction with microRNA-372 in liver cancer. Nucleic Acids Res 38: 5366-5383, 2010.

12. Wang J, Park JS, Wei Y, Rajurkar M, Cotton JL, Fan Q, Lewis BC, Ji $\mathrm{H}$ and Mao J: TRIB2 acts downstream of Wnt/TCF in liver cancer cells to regulate YAP and C/EBP $\alpha$ function. Mol Cell 51: 211-225, 2013.

13. Livak KJ and Schmittgen TD: Analysis of relative gene expression data using real-time quantitative PCR and the 2(-Delta Delta C(T)) Method. Methods 25: 402-408, 2001.

14. Johannessen M, Delghandi MP and Moens U: What turns CREB on? Cell Signal 16: 1211-1227, 2004.

15. van Dam H and Castellazzi M: Distinct roles of Jun: Fos and Jun: ATF dimers in oncogenesis. Oncogene 20: 2453-2464, 2001.

16. Benbrook DM and Jones NC: Heterodimer formation between CREB and JUN proteins. Oncogene 5: 295-302, 1990.

17. Mayr B and Montminy M: Transcriptional regulation by the phosphorylation-dependent factor CREB. Nat Rev Mol Cell Biol 2: 599-609, 2001.

18. Böhm M, Moellmann G, Cheng E, Alvarez-Franco M, Wagner S, Sassone-Corsi P and Halaban R: Identification of p90RSK as the probable CREB-Ser133 kinase in human melanocytes. Cell Growth Differ 6: 291-302, 1995.

19. Chrivia JC, Kwok RP, Lamb N, Hagiwara M, Montminy MR and Goodman RH: Phosphorylated CREB binds specifically to the nuclear protein CBP. Nature 365: 855-859, 1993.

20. Kundu TK, Palhan VB, Wang Z, An W, Cole PA and Roeder RG: Activator-dependent transcription from chromatin in vitro involving targeted histone acetylation by p300. Mol Cell 6: 551-561, 2000.

21. Smalley KS: A pivotal role for ERK in the oncogenic behaviour of malignant melanoma? Int J Cancer 104: 527-532, 2003.

22. Okazaki K and Sagata N: The Mos/MAP kinase pathway stabilizes c-Fos by phosphorylation and augments its transforming activity in NIH 3T3 cells. EMBO J 14: 5048-5059, 1995.

23. Murphy LO, Smith S, Chen RH, Fingar DC and Blenis J: Molecular interpretation of ERK signal duration by immediate early gene products. Nat Cell Biol 4: 556-564, 2002.

24. Mao L, Yang L, Tang Q, Samdani S, Zhang G and Wang JQ: The scaffold protein Homerlb/c links metabotropic glutamate receptor 5 to extracellular signal-regulated protein kinase cascades in neurons. J Neurosci 25: 2741-2752, 2005. 